\title{
SYNTHESIS OF ULTRASOUND FIELD SOURCES BASED ON PHASE SCREEN APPROXIMATION
}

\author{
Dmitry Sukhanov ${ }^{1}$, Ksenia Zavyalova, Nadezhda Erzakova \\ Tomsk State University, 634050, Tomsk, Russia
}

\begin{abstract}
Here is proposed the method for synthesizing the sources of an acoustic field on the basis of an approximation of the phase screen. The technology of manufacturing ultrasonic phased arrays providing the formation of a field of a given distribution is proposed. An experimental setup has been developed for the formation of a vortex field at a distance of $10 \mathrm{~cm}$.
\end{abstract}

\section{Introduction}

The formation of an ultrasonic field of the required distribution is an urgent task in the development of ultrasonic sounding and tomography systems, technologies of acoustic levitation and remote particle control, augmented reality devices for the transmission of tactile information [1]. The main direction of solving similar problems is the use of phased arrays $[2,3]$ of ultrasonic radiators (Figure 1). For the formation of the field, it is advisable to control the signal of each radiator separately, but such a scheme is rather complicated and expensive. A simpler solution is to use a single signal generator, which is fed through the delay lines to the emitters, thereby ensuring a phase difference between the elements. Since the ultrasonic signal is relatively low-frequency, too long electrical delay lines are required.

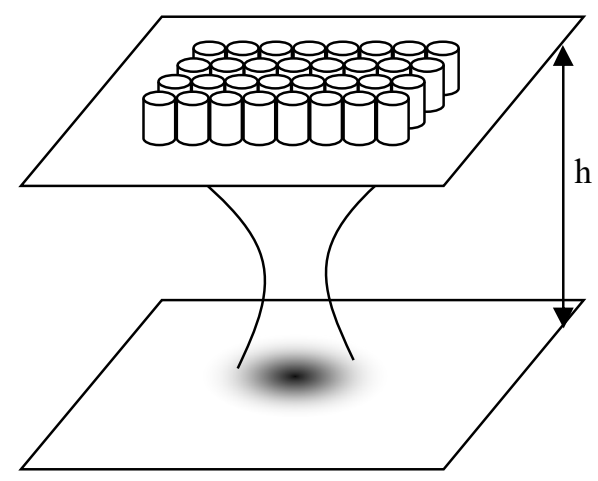

Fig.1. Scheme of field generation.

\footnotetext{
${ }^{1}$ Corresponding author: sdy@mail.tsu.ru
} 
It is proposed to use acoustic delay lines formed after each radiator. In this case, the length of the delay lines does not exceed the length of the ultrasonic wave, in this case, $8.25 \mathrm{~mm}$ at a frequency of $40 \mathrm{kHz}$ for a sound velocity in the air of $330 \mathrm{~m} / \mathrm{s}$.

\section{Field formation return task}

Let us consider the possibility of forming a vortex beam with the help of a grid of emitters placed in a hexagonal grid with a step of $11 \mathrm{~mm}$. In Figure 2 the required distribution of the field is shown, which must be created at a distance of $10 \mathrm{~cm}$. The required field distribution we define by following formula:

$$
U(x, y)=\exp (i \varphi-i k r / 2) \cdot\left(r \geq R_{1}\right) \cdot\left(r \leq R_{2}\right),
$$

where $r=\sqrt{x^{2}+y^{2}}, R_{1}=1 \mathrm{~cm}, R_{2}=5 \mathrm{~cm}$.

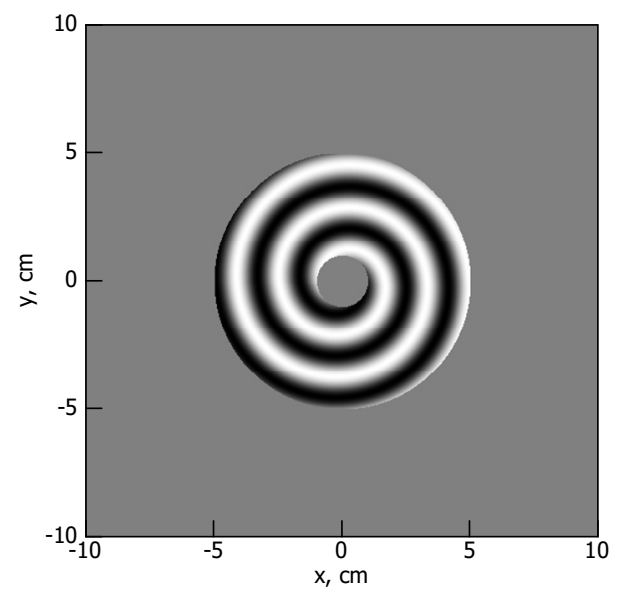

Fig.2. Required field distribution.

To obtain the amplitude-phase distribution of the sources from the target field, we use the wave reversal method $[4,5]$. We propose to reverse propagate only homogeneous waves. Let us solve this task by means of spatial plane wave spectrum representation:

$$
\tilde{U}\left(k_{x}, k_{y}\right)=\frac{1}{4 \pi^{2}} \int_{-\infty}^{\infty} \int_{-\infty}^{\infty} U(x, y) \exp \left(-i x k_{x}-i y k_{y}\right) d x d y .
$$

It is proposed to multiply the spatial spectrum of this field by $\exp \left(i k_{z} h\right)^{*}$, where ${ }^{*}$ - is a complex conjugation operation, $k_{z}=\sqrt{k^{2}-k_{x}^{2}-k_{y}^{2}}$ - is the projection of the wave vector on the axis perpendicular to the plane of the lattice, the wave number, and the $h$-distance from the array of radiators to the plane of the target field distribution. The plane wave spectrum of field in the plane of sources is:

$$
\widetilde{P}\left(k_{x}, k_{y}\right)=\tilde{U}\left(k_{x}, k_{y}\right) \cdot\left(\exp \left(i k_{z} h\right)\right)^{*},
$$

Reconstruction of field distribution if the source plane is: 


$$
P(x, y)=\int_{-\infty}^{\infty} \int_{-\infty}^{\infty} \widetilde{P}\left(k_{x}, k_{y}\right) \exp \left(i x k_{x}+i y k_{y}\right) d k_{x} d k_{y} .
$$

That is, using the inversion of waves in the frequency domain, the required amplitudephase distribution is calculated on a given plane. Formula (3) allows to calculate homogeneous waves and to avoid enhancement of heterogeneous waves. For the case shown on Figure 2, it was calculated required distribution of sources field on $40 \mathrm{kHz}$ (Figure 3).

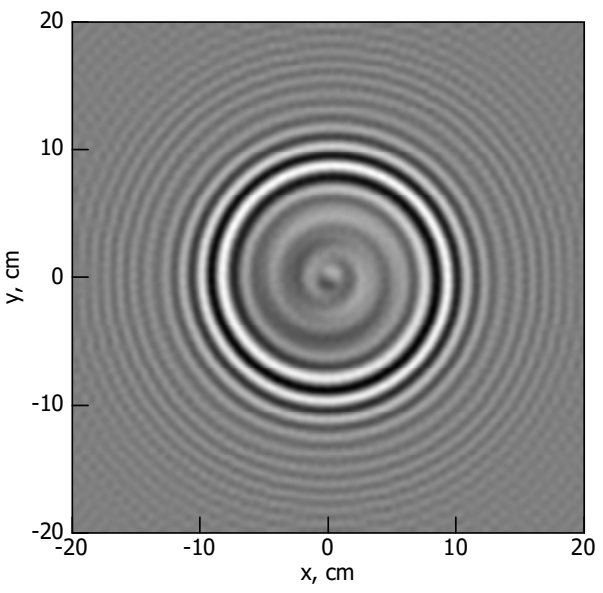

a

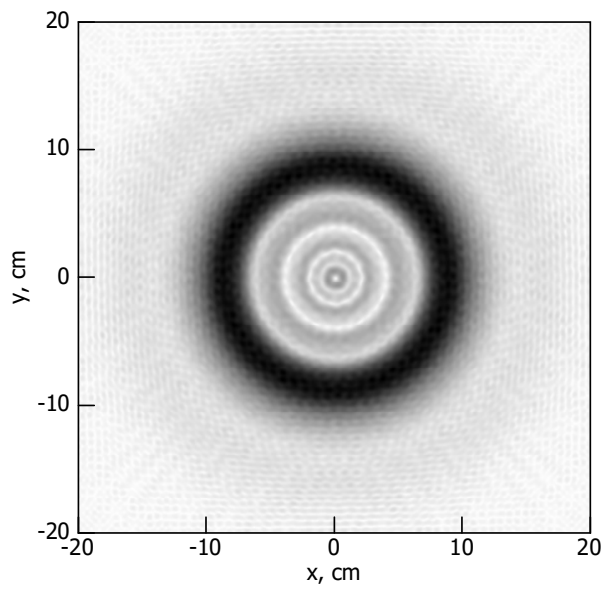

b

Fig.3. Reconstruction of sources distribution: $\mathrm{a}$ - field of sources; $\mathrm{b}$ - magnitude of sources field.

\section{Sources placement and numerical simulation}

We can see that the maximum magnitudes of sources is concentrated in a narrow circle area. That is why it is possible to place sources only in this area to minimize a number of elements. The most dense placement of circular elements on a plane is a cellular hexagonal placement. We propose to approximate the circle by hexagon of transmitters, as shown on Figure 4. On Figure $4 b$ the phase of sources is shown on grayscale picture. The magnitude of each source is the same.
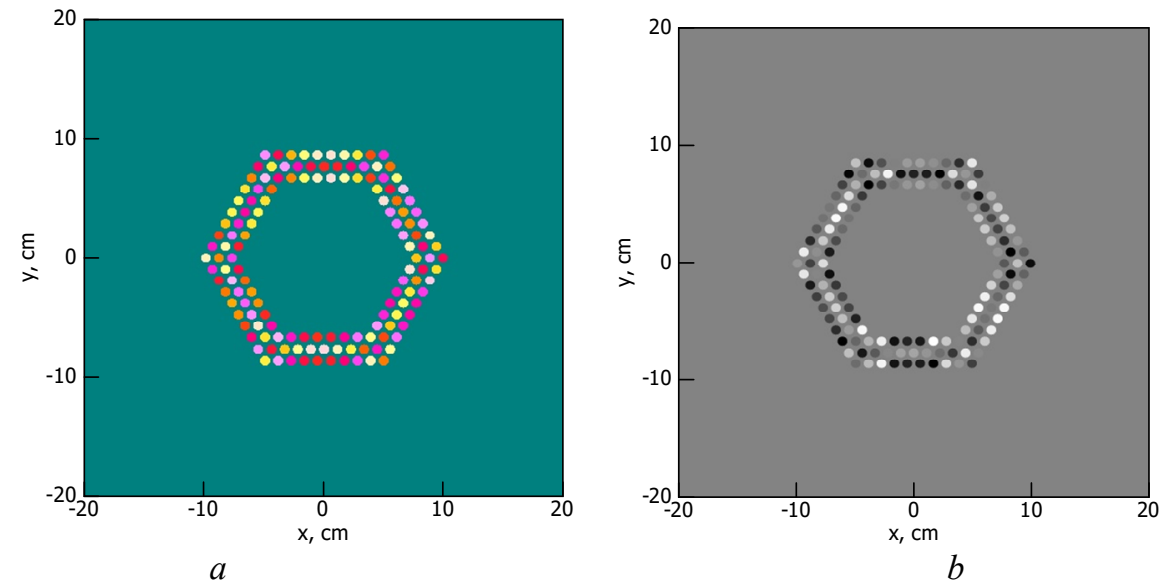

Fig.4. Placement of elements (a) and phase distribution of sources (b). 
In Figure $4 a$ shows the result of the calculation of signals in the emitters, where each color channel corresponds to: red - amplitude; green - cosine quadrature; blue is a sine quadrature.

To calculate the forward field we use the same approach we used for sources reconstruction, but in forward direction:

$$
\begin{aligned}
& \widetilde{B}\left(k_{x}, k_{y}\right)=\widetilde{P}\left(k_{x}, k_{y}\right) \cdot\left(\exp \left(i k_{z} h\right)\right) \\
& B(x, y)=\int_{-\infty}^{\infty} \int_{-\infty}^{\infty} \widetilde{B}\left(k_{x}, k_{y}\right) \exp \left(i x k_{x}+i y k_{y}\right) d k_{x} d k_{y}
\end{aligned}
$$

After calculating the direct propagation (5--6) of the field in the spatial spectra, the expected distribution in the target plane was obtained (Figure 5). It can be seen that the field has a vortex character, as expected, but there are side lobes, which is due to the sparseness of the grating.

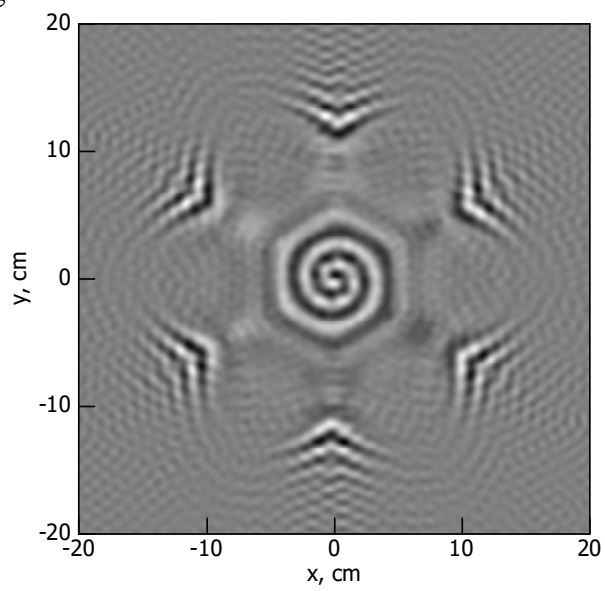

Fig.5. Numerical modeling of field in the focusing plane.

A plurality of in-phase point emitters located in a hexagonal grid with a pitch of $11 \mathrm{~mm}$ are set at a distance from the median plane, so that this distance provides the required phase shift, based on the approach of the phase screens.

\section{Experimental studies}

The experimental setup is made of 144 emitters installed in a plate with holes of the calculated depth (Figure 6). As a result of measuring the plane distribution of the field at a range of $10 \mathrm{~cm}$ (Figure 7), it was found that it corresponds with the numerical model qualitatively. 


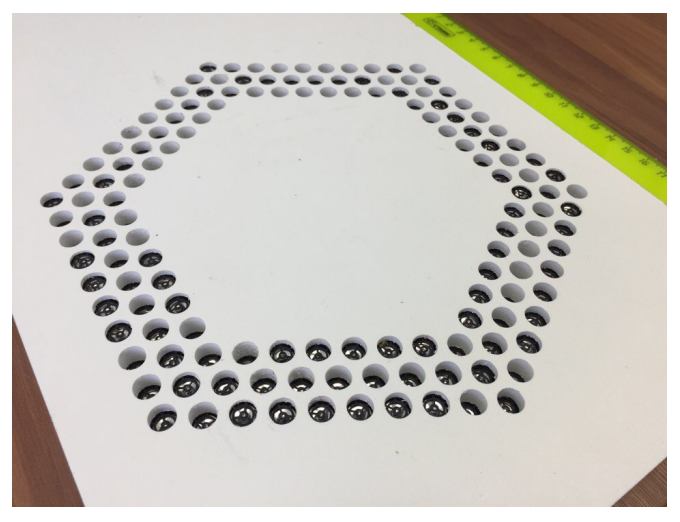

Fig.6. Photography of the phased array.

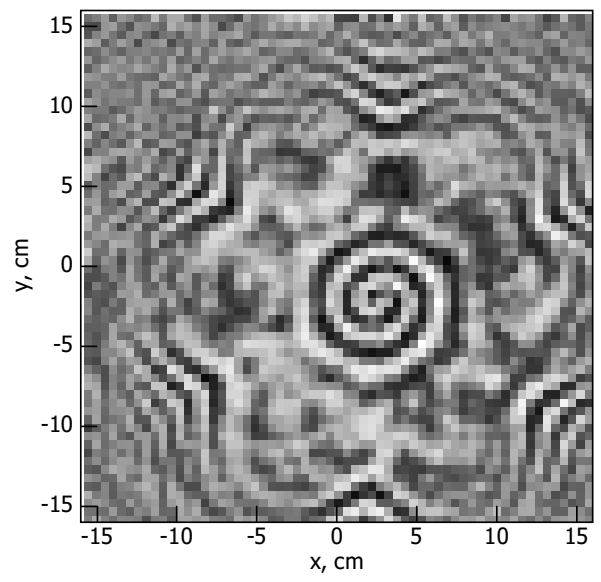

Fig.7. Measured field.

\section{Conclusion}

A method is proposed for synthesizing ultrasonic field sources in air to form a given field distribution. The possibility of its application has been shown numerically and experimentally.

\section{Acknowledgments}

The scientific research was carried out with the support of the grant of the Russian Science Foundation No. 17-79-20051.

\section{References}

[1] S. Hashizume, A. Koike, T. Hoshi, Y. Ochiai, ACM SIGGRAPH 2017 Posters, No a57, 129315 (2017)

[2] A.I. Korobov, M.Y. Izosimova, S.A. Toschov, Physics Procedia 3, 201 (2010)

[3] A. Ganguli, R.X. Gao, K. Liang, J. Jundt, A. Ordonez, IEEE Transactions on Instrumentation and Measurement 59, 5353739 (2010)

[4] Y. Jing, F.C. Meral, G.T. Clement, Physics in Medicine and Biology 57, 901 (2012)

[5] H. Nicacio, V.L.S.N. Button, R.G. Dantas, E.T. Costa, Proceedings of the IEEE Ultrasonics Symposium 1, 559 (2002) 